\title{
A RESULT ABOUT THE HILBERT TRANSFORM ALONG CURVES
}

\author{
LINDA V. SAAL
}

(Communicated by J. Marshall Ash)

\begin{abstract}
Let $G$ be a connected and simply connected, nilpotent Lie group and let $\gamma:(-1,1) \rightarrow G$ be a (connected) analytic curve such that $\gamma(0)=0$. Then the Hilbert transform along $\gamma$,

$$
T f(x)=p \cdot v \cdot \int_{0<|t|<1} f\left(x \gamma(t)^{-1}\right) d t / t,
$$

is bounded on $L^{p}(G), 1<p<\infty$.
\end{abstract}

\section{INTRODUCTION}

Let $G$ be a simply connected, nilpotent Lie group. From [Ch] we know that if $\gamma$ is a homogeneous curve with respect to a group of dilations of $G, \gamma(0)=$ 0 , then the Hilbert transform along $\gamma$ is a bounded operator on $L^{p}(G)$, for $1<p<\infty$; moreover $T f(x)$ exists a.e. for all $f \in L^{p}(G)$. In [RS2] singular integral operators whose kernels are homogeneous distributions of critical degree with mean value zero and supported on a homogeneous, analytic submanifold of $G$ are considered.

The purpose of this note is to prove that if $\gamma:(-1,1) \rightarrow G$ is a connected, analytic curve such that $\gamma(0)=0$ then the

$$
T f(x)=p . v . \int_{0<|t|<1} f\left(x \gamma(t)^{-1}\right) d t / t
$$

is a bounded operator on $L^{p}(G), 1<p<\infty$.

For the proof we first assume that a certain group of dilations associated to $\gamma$ are automorphisms of $G$ and also that $\gamma$ generates $G$. We use the iteration argument of [Ch]. The general case follows from a transference theorem [RS2].

Finally the boundedness of the maximal operator

$$
T^{*} f(x)=\sup _{r>0}\left|\int_{r<|t|<1} f\left(x \gamma(t)^{-1}\right) d t / t\right|
$$

on $L^{p}(G)$, implies that $T f(x)$ exists a.e. for all $f \in L^{p}$.

Received by the editors January 10, 1989 and, in revised form, July 1, 1989.

1980 Mathematics Subject Classification (1985 Revision). Primary 42B20.

This research was supported by the Consejo Nacional de Investigaciones Cientificas y Técnicas, Argentina. 
The proof of the theorem. Let $G$ be a connected and simply connected, nilpotent Lie group. Let $\gamma:(-1,1) \rightarrow G$ be a (connected) analytic curve that generates $G$ in the sense that $\gamma$ is not contained in any proper closed subgroup of $G$. Also assume that $\gamma(0)$ is the identity of $G$. Denote by exp: $g \rightarrow G$ the exponential map. First of all we remark the following facts:

(i) $\gamma$ generates $G$ if and only if $\left\{d^{j} \bar{\gamma} /\left.d t^{j}\right|_{t=0}\right\}_{j \in N}$ generate $g$ as a Lie algebra, with $\bar{\gamma}=\gamma_{\circ} \exp ^{-1}$. Indeed, let $h$ be the Lie subalgebra of $g$ generated by $\left\{d^{j} \bar{\gamma} /\left.d t^{j}\right|_{t=0}\right\}$ and $H=\exp h$. Since $\bar{\gamma}$ is analytic, $\bar{\gamma} \subset h$.

(ii) We can choose a coordinate system on $G$ such that

$$
\gamma(t)=\left(\frac{t^{a_{1}}}{a_{1} !} \varphi_{1}(t), \ldots, \frac{t^{a_{k}}}{a_{k} !} \varphi_{k}(t), 0, \ldots, 0\right)
$$

with $1 \leq a_{1}<a_{2}<\cdots<a_{k}, \varphi_{i}$ are analytic functions and $\varphi_{i}(0)=1$. Indeed, following [SW], we choose $a_{1}=\inf \left\{j\left|d^{j} \bar{\gamma} / d t^{j}\right|_{t=0} \neq 0\right\}$. Given $a_{1}, \ldots, a_{i}$, define

$$
\begin{array}{r}
a_{i+1}=\inf \left\{j \mid j>a_{i} \text { and }\left\{\left.\frac{d^{a_{1}} \bar{\gamma}}{d t^{a_{1}}}\right|_{t=0}, \ldots,\left.\frac{d^{a} \bar{\gamma}}{d t^{a_{i}}}\right|_{t=0},\left.\frac{d^{j} \bar{\gamma}}{d t^{j}}\right|_{t=0}\right\}\right. \\
\text { are linearly independent }\} .
\end{array}
$$

Define $e_{i}=d^{a_{i}} \bar{\gamma} /\left.d t^{a_{i}}\right|_{t=0}$. We thus obtain a set $\left\{e_{1}, \ldots, e_{k}\right\}, k \leq n$, maximal with respect to be L.I. such that

$$
\bar{\gamma}(t)=\frac{t^{a_{1}}}{a_{1} !} \varphi_{1}(t) e_{1}+\cdots+\frac{t^{a_{k}}}{a_{k} !} \varphi_{k}(t) e_{k} .
$$

We extend $\left\{e_{1}, \ldots, e_{k}\right\}$ to a basis of $g,\left\{e_{1}, \ldots, e_{k}, e_{k+1}, \ldots, e_{n}\right\}$. If $A$ denotes the change of basis matrix, the coordinates of $\gamma(t)$ with respect to $\psi=\exp \circ A$ are $\left(\left(t^{a_{1}} / a_{1} !\right) \varphi_{1}(t), \ldots,\left(t^{a_{k}} / a_{k} !\right) \varphi_{k}(t), 0, \ldots, 0\right)$. From now on we fix this coordinate system on $G$.

(iii) We now associate to $\gamma$ a group of dilations $D_{r}, r>0$, by

$$
D_{r}\left(x_{1}, \ldots, x_{n}\right)=\left(r^{a_{1}} x_{1}, \ldots, r^{a_{k}} x_{k}, r^{a_{k+1}} x_{k+1}, \ldots, r^{a_{n}} x_{n}\right) \text {, }
$$

where $a_{1}, \ldots, a_{k}$ are as in (ii) and $a_{k+1}, \ldots, a_{n}$ are arbitrary integers. Let $Q=a_{1}+\cdots+a_{n}$.

(iv) Let $\gamma_{0}$ be the curve in $G$ with coordinates

$$
\left(t^{a_{1}} / a_{1} !, \ldots, t^{a_{k}} / a_{k} !, 0, \ldots, 0\right) .
$$

Then $\gamma_{0}$ is analytic, homogeneous with respect to $D_{r}$ and generates $G$. Indeed,

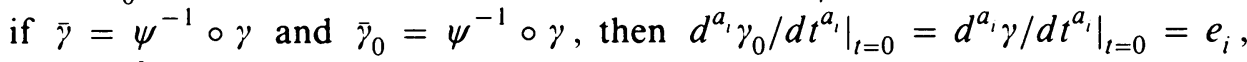
$1 \leq i \leq k$.

We have associated to $\gamma$ a group of dilations $\left\{D_{r}\right\}_{r>0}$ and a curve $\gamma_{0}$ that is homogeneous with respect to $D_{r}$ and also generates $G$. For the moment we also assume that $\left\{D_{r}\right\}$ are automorphisms of $G$ and fix a homogeneous norm 
with respect to $D_{r}$, e.g., $|x|=\max _{1 \leq i \leq n}\left\{\left|x_{i}\right|^{1 / a_{i}}\right\}$. Denote by $\bar{B}$ the closure of the unit ball in $\mathbf{R}^{n}$.

Lemma 1. Let $\phi, \phi_{0}$ be the functions on $\bar{B}$ defined by $\phi\left(t_{1}, \ldots, t_{n}\right)=$ $\gamma\left(t_{1}\right) \ldots \gamma\left(t_{n}\right) ; \phi_{0}\left(t_{1}, \ldots, t_{n}\right)=\gamma_{0}\left(t_{1}\right) \ldots \gamma_{0}\left(t_{n}\right)$. For each $j \in \mathbf{N}, j \geq 0$, define $\phi_{j}$ on $\left\{t \in \mathbf{R}^{n}\left|\frac{1}{2} \leq\right| t_{i} \mid \leq 1\right\}$ by $\phi_{j}(t)=D_{2^{\prime}} \phi\left(2^{-j} t\right)$. If $J_{j}(t)=\operatorname{det} d \phi_{j}(t)$ and $J_{0}(t)=\operatorname{det} d \phi_{0}(t)$, then $J_{j}(t) \underset{j \rightarrow+\infty}{\longrightarrow} J_{0}(t)$ uniformly on $\left\{t \in \mathbf{R}^{n}\left|\frac{1}{2} \leq\right| t_{i} \mid \leq 1\right\}$.

Proof. Let $\bar{\phi}_{j}(t)=\exp ^{-1} \phi_{j}(t), \bar{\phi}_{0}(t)=\exp ^{-1} \phi_{0}(t)$.

$$
\begin{aligned}
\bar{\phi}_{j}(t) & =\exp ^{-1}\left(D_{2^{j}} \phi\left(2^{-j} t\right)\right)=D_{2^{j}} \exp ^{-1} \phi\left(2^{-j} t\right) \\
& =D_{2^{j}} \exp ^{-1}\left(\gamma\left(2^{-j} t_{1}\right) \cdots \gamma\left(2^{-j} t_{n}\right)\right) .
\end{aligned}
$$

Then

$$
\bar{\phi}_{j}(t)=D_{2^{j}}\left(\bar{\gamma}\left(2^{-j} t_{1}\right)+\cdots+\bar{\gamma}\left(2^{-j} t_{n}\right)\right)+D_{2^{\prime}}\left(P_{1}(\cdots), \ldots, P_{n}(\cdots)\right)
$$

where $P_{j}(\cdots)$ denotes $P_{j}\left(\bar{\gamma}\left(2^{-j} t_{1}\right), \ldots, \bar{\gamma}\left(2^{-j} t_{n}\right)\right)$ and $P_{j}$ are polynomial functions on the coordinates of $\gamma\left(t_{1}\right), \ldots, \gamma\left(t_{n}\right)$.

If $\gamma$ is homogeneous, $\bar{\phi}_{j}(t)=\bar{\phi}(t)$ and the first term of the right side of $(*)$ is also independent of $j$. Thus either $P_{l} \equiv 0$ or $P_{l}\left(t_{1}, \ldots, t_{n}\right)$ is homogeneous of degree $a_{l}, l=1, \ldots, n$,

$$
\begin{aligned}
& P_{l}\left(t_{1}, \ldots, t_{n}\right)=\sum \beta_{j_{1}, j_{k}}^{i_{1}, i_{k}} \varphi_{j_{1}}\left(t_{i_{1}}\right) \cdots \varphi_{j_{k}}\left(t_{i_{k}}\right) t_{i_{1}}^{a_{j_{1}}} \cdots t_{i_{k}}^{a_{j_{k}}} \\
& =\sum \beta_{j_{1},}^{i_{1}, i_{k}} F_{j_{1}}, \ldots, j_{k}\left(t_{i_{1}}, \quad, t_{i_{k}}\right) t_{i_{1}}^{a_{j_{1}}} \cdots t_{i_{k}}^{a_{j_{k}}},
\end{aligned}
$$

with $a_{j_{1}}+\cdots+a_{j_{k}}=a_{l}, \beta_{j_{1}}^{i_{1},}, i_{j_{k}} \in \mathbf{C}$ and $F_{j_{1}}, \ldots, j_{k}(0)=1$. Then

$$
\begin{aligned}
\bar{\phi}_{j}(t)= & \left(\ldots, \sum_{i=1}^{n} \varphi_{l}\left(2^{-j} t_{i}\right) t_{i}^{a_{l}}, \ldots\right) \\
& +\left(\ldots, \sum \beta^{s} F_{j_{1}, j_{k}}\left(2^{-j} t_{i_{1}}, \ldots, 2^{-j} t_{i_{k}}\right) t_{i_{1}}^{a_{j_{1}}} \ldots t_{i_{k}}^{a_{j_{k}}}, \ldots\right) .
\end{aligned}
$$

By the mean value theorem applied to $\varphi_{l}^{\prime s}$ and $F_{j_{1}, j_{k}}^{\prime s}$, we have that

$$
\begin{aligned}
\bar{\phi}_{j}(t)= & \left(\ldots, \sum_{i=1}^{n} t_{i}^{a_{1}}+\sum \beta^{\prime s} t_{i_{1}}^{a_{j_{1}}} \ldots t_{i_{k}}^{a_{J_{1}}}, \ldots\right) \\
& +2^{-j}\left(G_{1}\left(t_{1}, \ldots, t_{n}\right), \ldots, G_{n}\left(t_{1}, \ldots, t_{n}\right)\right) \\
= & \bar{\phi}_{0}(t)+2^{-j}\left(G_{1}\left(t_{1}, \ldots, t_{n}\right), \ldots, G_{n}\left(t_{1}, \ldots, t_{n}\right)\right),
\end{aligned}
$$

with $G_{k}$ bounded on $\left\{t\left|\frac{1}{2} \leq\right| t_{i} \mid \leq 1\right\}, k=1, \ldots, n$. This proves the lemma since $\operatorname{det} d_{x} \exp =1$.

Remark. (v) If $\gamma$ is an analytic curve that generates $G$ then $\phi: \bar{B} \rightarrow G$ given by $\phi\left(t_{1}, \ldots, t_{n}\right)=\gamma\left(t_{1}\right) \cdots \gamma\left(t_{n}\right)$ has rank $n$ almost everywhere. Indeed, since $\gamma_{0}$ is a homogeneous curve that generates $G, J_{0} \not \equiv 0[\mathrm{Ch}]$. Since $\gamma_{0}$ is analytic, 
$J_{0}(t) \neq 0$ almost everywhere. Let $J(t)=\operatorname{det} \phi(t)$. Since $\phi_{j}(t)=D_{2^{j}} \phi\left(2^{-j} t\right)$, $2^{j(Q-n)} J\left(2^{-j} t\right)=J_{j}(t) \underset{j \rightarrow \infty}{\longrightarrow} J_{0}(t)$ on $\left\{t\left|\frac{1}{2} \leq\right| t_{i} \mid \leq 1\right\}$. Then $J \not \equiv 0$. (Furthermore $J(t) \neq 0$ almost everywhere).

By a result of [RS2] we know that if $\phi: \bar{B} \rightarrow \mathbf{R}^{n}$ is an analytic function having rank $n$ at almost everywhere point and $\psi$ is a $C^{1}$-function supported on $\bar{B}$ then the transported measure by $\phi$ of $\psi(t) d t$ is absolutely continuous and its density $\rho$ satisfies and $L^{1}$-Hölder condition $\int|\rho(x+y)-\rho(x)| d x \leq c\|y\|^{\delta}$, for some $\delta>0$ depending on $\phi, c>0$ that depends also on $\psi$.

In the next lemma we say a little more about $c$.

Lemma 2. Let $J(t)=\operatorname{det} d \phi(t)$. Then

$$
\int|\rho(x+y)-\rho(x)| d x \leq \widetilde{C}\|y\|^{\delta}\left(\int_{\bar{B}}|\nabla \psi|+|\psi|\right)^{\delta}\left(\int_{\bar{B}}|\psi| / 2 \delta /|J|(1-\delta)\right)^{1-\delta},
$$

for some $\delta>0, \widetilde{\mathbf{C}}>0$, depending only on $\phi$.

Proof. Following the proof of [RS2] we denote by $Z$ the set of zeros of $J(t)$ and consider a covering $\left\{B_{j}\left(z_{j}, r_{j}\right)\right\}_{j}$ of $\bar{B}-Z$, satisfying,

(1) $r_{j}=c_{0}\left|J\left(z_{j}\right)\right|$;

(2) the balls $B_{j}^{*}=B\left(z_{j}, 2 r_{j}\right)$ are disjoint from $Z$ and have the bounded overlapping property; i.e. there is an integer $N$ such that no point belongs to more that $N$ of the $B_{j}^{*}$.

Let $\left\{n_{j}\right\}$ be a smooth partition of unity on $\bar{B}-Z$ subordinated to $\left\{B_{j}\right\}$ and such that $\left\|\nabla n_{j}\right\|_{\infty} \leq \widetilde{\mathbf{C}} r_{j}^{-1}$. Let $\psi_{j}=\psi n_{j}$ and let $\rho_{j}$ be the transported measure by $\phi$ of $\psi_{j}$. Since $\left.\phi\right|_{B_{j}}$ is invertible [RS2] $\rho_{j}(x)=\left|J \phi^{-1}(x)\right|^{-1} \psi_{j}\left(\phi^{-1}(x)\right)$ and thus

$$
\int\left|\rho_{j}(x)\right| d x=\left\|\psi_{j}\right\|_{1}=\int\left|\psi_{j}\right|
$$

and

$$
\begin{aligned}
& \int\left|\nabla \rho_{j}(x)\right| d x \\
& \leq \widetilde{\mathbf{C}}\left(\int|\nabla \psi(t)| n_{j}(t)|J(t)|^{-1} d t+\int|\psi|\left|\nabla n_{j}\right||J(t)|^{-1} d t+\int|J(t)|^{-2}|\psi| d t\right) \\
& \leq \widetilde{\mathbf{C}} r_{j}^{-2}\left(\int_{B_{j}}|\nabla \psi|+\int_{B_{j}}|\psi|\right) .
\end{aligned}
$$

This implies that if $0<\delta \leq 1$,

$$
\int\left|\rho_{j}(x+y)-\rho_{j}(x)\right| d x \leq \widetilde{\mathbf{C}}\|y\|^{\delta} r_{j}^{-2 \delta}\left(\int_{B_{j}}|\nabla \psi|+|\psi|\right)^{\delta}\left(\int_{B_{j}}|\psi|\right)^{1-\delta} .
$$


Now by Hölder's inequality

$$
\begin{aligned}
& \sum_{j}\left(\int_{B_{j}}|\nabla \psi|+|\psi|\right)^{\delta} r_{j}^{-2 \delta}\left(\int_{B_{j}}|\psi|\right)^{1-\delta} \\
& \quad \leq\left(\sum_{j}\left(\int_{B_{j}}|\nabla \psi|+|\psi|\right)\right)^{\delta}\left(\sum_{j} r_{j}^{-2 \delta /(1-\delta)} \int_{B_{j}}|\psi|\right)^{1-\delta} .
\end{aligned}
$$

The lemma follows from the fact that $\left.J\right|_{B_{j}^{*}}$ is comparable with $r_{j}$ and from the bounded overlapping property.

Theorem. Let $\gamma$ be an analytic curve that generates $G$ and such that the group of dilations associated to $\gamma$ are automorphisms of $G$. Then

$$
T f(x)=p . v . \int_{0<|t|<1} f\left(x \gamma(t)^{-1}\right) d t / t
$$

defines a bounded operator on $L^{P}(G), 1<p<\infty$.

Proof. (We follow [RS2] for the proof.) Denoted by $\mu$ the distribution given by $\mu(f)=p . v . \int_{0<|t| \leq 1} f(\gamma(t)) d t / t$. Let $\tilde{\varphi}$ be in $\mathscr{C}^{\infty}\left(\frac{1}{2}, 2\right)$ such that $\sum_{j=0} \tilde{\varphi}\left(2^{j}|t|\right)$ $=1$. Let $\varphi(x)=\varphi(|x|)$ where || denote the homogeneous norm defined in Remark (iv). Let $\mu_{j}=\tilde{\varphi}\left(2^{j}|t|\right) \mu$.

Let $\varphi_{j}(x)=2^{j Q} \varphi\left(D_{2^{j}} x\right)$ and $\eta_{j}=\frac{1}{c}\left(\varphi_{j+1}-\varphi_{j}\right)$ with $c=\int \varphi$.

Since $\left\{c^{-1} \varphi_{j}\right\}$ is an approximate identity in $G$ we can write

$$
\delta=c^{-1} \lim _{j \rightarrow+\infty} \varphi_{j}=\sum_{j \geq j_{0}} \eta_{j}+c^{-1} \varphi_{j_{0}}
$$

for each $j_{0}$ fixed. Also $\eta=\sum_{j \geq 0} \mu_{j}$. Then

$$
\mu=\delta * \sum_{j} \mu_{j}=\sum_{j}\left(\sum_{k \geq j_{0}} \eta_{k} * \mu_{j}\right)+c^{-1} \sum_{j} \varphi_{j_{0}} * \mu_{j} .
$$

For each $j$ fixed, take $j_{0}=j+1$. Then

$$
\begin{aligned}
\mu & =\sum_{k \geq 1}\left(\sum_{j \geq 0} \eta_{k+j} * \mu_{j}\right)+c^{-1} \sum_{j}\left(\varphi_{j+1} * \mu_{j}\right) \\
& =\sum_{k \geq 1} M_{k}+N .
\end{aligned}
$$

If we prove

(1) For $0<\epsilon \leq 1,\left\|M_{k}\right\|_{p, p} \leq \mathbf{C}_{\epsilon, p} 2^{\epsilon k},\|N\|_{p, p} \leq \mathbf{C}$.

(2) $\exists \sigma>0$ such that $\left\|M_{k}\right\|_{2,2} \leq \mathbf{C} 2^{-\sigma k}$.

Then the theorem follows by interpolation and duality. Indeed, fix $1<p<2$ and take $1<p_{0}<p$. Then $\frac{1}{p}=\frac{1}{2} s+(1-s) \frac{1}{p_{0}}$ for some $0<s<1$. Thus 
$\left\|M_{k}\right\|_{p, p} \leq \mathbf{C}_{\epsilon} 2^{-\sigma k s} 2^{\epsilon k(1-s)}$ for $0<\epsilon \leq 1$ and some $\sigma>0$. Choosing $\epsilon$ small enough we obtain $\sum_{k}\left\|M_{k}\right\|_{p, p}<+\infty$.

(1) We observe that $M_{k}=\sum_{j \geq 0} \eta_{k+j} * \mu_{j}=\sum_{j \geq 0} f_{j}^{k}$ where $f_{j}^{k}$ has the following properties

(a) $f_{j}^{k}=0$,

(b) $\int\left|f_{j}^{k}(x y)-f_{j}^{k}(x)\right| d x \leq \mathbf{C} 2^{(k+j) \epsilon}|y|^{\epsilon}$,

(c) $\sup f_{j}^{k} \subset\left\{x|| x\left|\leq \mathbf{C} 2^{-j}\right|\right)$,

(d) $\int\left|f_{j}^{k}\right| \leq \mathbf{C}$.

Then Cotlar's lemma implies that $\left\|M_{k}\right\|_{2,2} \leq \mathbf{C} 2^{\epsilon k}$ and the weak type (1.1) of $M_{k}$ follows by checking that $\int_{|x|>2|y|}\left|M_{k}(x y)-M_{k}(x)\right| d x \leq \mathbf{C} 2^{k \epsilon}$. The same argument holds for $N$. For a proof see [ChNSW].

(2) By Cotlar's lemma it is enough to prove that for some $\sigma>0$ $\left\|f_{j}^{k} * f_{l}^{k}\right\|_{2,2} \leq \mathbf{C} 2^{-\sigma k} 2^{-|j-l| \sigma}$. We check this for $j>l$, by using the iteration argument of $[\mathrm{Ch}]$.

$$
\begin{aligned}
\left\|f_{j}^{k} * f_{l}^{k}\right\|_{2,2} & \leq \mathbf{C}\left\|\eta_{k+j} * \mu_{j} * \mu_{l}^{*}\right\|_{2,2} \\
& \leq \mathbf{C}\left\|\eta_{k+j} * \mu_{j}\right\|^{1 / 2}\left\|\mu_{l}^{*} * \mu_{l} * \mu_{j}^{*} * \eta_{k+j}\right\|^{1 / 2} \\
& \leq \cdots \leq \mathbf{C}\left\|\eta_{k+j} * \mu_{j}\right\|^{1-2^{-r}}\left\|\left(\mu_{l}^{*} * \mu_{l}\right)^{2^{r-1}} * \mu_{j}^{*} * \eta_{k+j}\right\|^{2^{-r}} .
\end{aligned}
$$

Let $\psi_{l}\left(t_{1}, \ldots, t_{n}\right)=\prod_{i=1}^{n} \frac{1}{t_{i}} \tilde{\varphi}\left(2^{l} t_{i}\right)$ and $\phi\left(t_{1}, \ldots, t_{n}\right)=\gamma\left(t_{1}\right) \cdots \gamma\left(t_{n}\right)$ as in Lemma 1.

Since $\mu_{l} * \cdots * \mu_{l}$ is the transported measure by $\phi$ of $\psi_{l}(t) d t$ and since $\phi$ is analytic with rank $n$ almost everywhere, $\mu_{l} * \cdots * \mu_{l}$ is absolutely continuous [RS2].

If $\rho_{l}$ denotes its density, we have to prove that

$$
\left\|\rho_{l} * \mu_{j}^{*} * \eta_{k+j}\right\|_{1} \leq \mathbf{C} 2^{-\sigma k} 2^{-|j-l| \sigma}
$$

for some $\sigma>0$. (Recall that we have assumed $l<j$.)

Let $\tilde{\rho}_{l}(x)=2^{-l Q} \rho_{l}\left(D_{2}-l x\right)$. Then $\tilde{\rho}_{l}$ is the transported measure by $\phi_{l}(t)=$ $D_{2^{\prime}} \phi\left(2^{-l} t\right)$ of $\tilde{\psi}(t)=2^{-\ln } \psi_{l}\left(2^{-l} t\right) \quad\left(\sup \tilde{\psi} \subset\left\{t\left|\frac{1}{2} \leq\right| t_{i} \mid \leq 2\right\}\right)$.

If we prove that

$$
\text { (A) } \int_{G}\left|\tilde{\rho}_{l}(x y)-\tilde{\rho}_{l}(x)\right| d x \leq \mathbf{C}|y|^{\epsilon}, \quad \text { for some } \mathbf{C}, \epsilon>0 \text {, independent of } l
$$

then $\int_{G}\left|\rho_{l}(x y)-\rho_{l}(x)\right| d x \leq \mathbf{C} 2^{l \epsilon}|y|^{\epsilon}$. From this and the fact that $\eta_{k+j}$ has mean value zero and sop $\eta_{k+j} \subset\left\{x|| x \mid \leq C 2^{-(k+j)}\right\}$ we obtain the inequality desired.

But if $\bar{\rho}_{l}=\tilde{\rho}_{l} \circ \exp$ and $\bar{\phi}_{l}=\exp ^{-1} \circ \phi_{l}$ then $\bar{\rho}_{l}$ is the transported measure of $\tilde{\psi}(t) d t$ by $\bar{\phi}$, and (A) is equivalent to

(B)

$$
\int_{\mathbf{R}^{n}}\left|\bar{\rho}_{l}(x+y)-\bar{\rho}_{l}(x)\right| d x \leq \mathbf{C}\|y\|^{\delta}, \quad \mathbf{C}, \delta>0 \text {, independent of } l \text { (see [RS2]). }
$$


Finally we prove (B). Since by Lemma 2 ,

$$
\int_{\mathbf{R}^{n}}\left|\bar{\rho}_{l}(x+y)-\bar{\rho}_{l}(x)\right| d x \leq \mathbf{C}\|y\|^{\delta}\left(\int|\nabla \tilde{\psi}|+|\tilde{\psi}|\right)^{\delta}\left(\int|\tilde{\psi}| /\left|\bar{J}_{l}\right| 2 \delta / 1-\delta\right)^{1-\delta}
$$

for some $0<\delta<1$, we only have to check that $\int_{\text {sup } \dot{\psi}}\left|\bar{J}_{l}^{(t)}\right|^{-\alpha} d t \leq \mathbf{C}$, with C independent of $l$, for some $0<\alpha<1$. By Lemma $1,2^{l(Q-n)} \bar{J}\left(2^{-l} t\right)=$ $\bar{J}_{l}(t)$ converges uniformly to $\bar{J}_{0}(t)$, which is a homogeneous polynomial of degree $Q-n$. Thus if we develop $\bar{J}(t)$ about 0 in the Taylor expansion, $\bar{J}(t)=P(t)+R(t)$, where $P$ is a homogeneous polynomial of degree $Q-n$ and $\frac{R(t)}{|t|^{Q-n}} \rightarrow_{|t| \rightarrow 0} 0$. By a linear change of variables we can normalize $P$ in the $t_{n}$-direction, i.e., $P(t)=t_{n}^{Q+n}+\sum_{j=0}^{Q-n-1} b_{j}\left(t_{1}, \ldots, t_{n-1}\right) t_{n}^{j}$ with $b_{j}(0)=0$. The Weierstrass preparation theorem now implies that

$$
\bar{J}(t)=\left(t_{n}^{Q-n}+a_{1}\left(t^{\prime}\right) t_{n}^{Q-n-1}+\cdots+a_{Q-n}\left(t^{\prime}\right)\right) h(t), \quad t^{\prime}=\left(t_{1}, \ldots, t_{n-1}\right),
$$

where $a_{1}, \ldots, a_{Q-n}$ and $h$ are analytic functions in a neighborhood of 0 , and $h(0) \neq 0$. (See $[\mathrm{H}]$.) Then

$$
\begin{gathered}
\int_{\sup \dot{\psi}}\left|\bar{J}_{l}(t)\right|^{-\alpha} d t=2^{-l(Q-n) \alpha} \int_{\sup \dot{\psi}}\left|\bar{J}\left(2^{-l} t\right)\right|^{-\alpha} d t \\
\leq \mathbf{C} 2^{-l(Q-n) \alpha} \int_{\frac{1}{2} \leq|t| \leq 2} 2^{l(n-Q) \alpha} \mid t_{n}^{Q-n}+2^{l} a_{1}\left(t^{\prime}\right) t_{n}^{Q-n-1} \\
+\cdots+\left.2^{l(Q-n)} a_{Q-n}\left(t^{\prime}\right)\right|^{-\alpha} d t_{n} d t^{\prime} \\
\quad \leq \mathbf{C} A_{\alpha} \int_{1 \leq\left|t^{\prime}\right| \leq 2}\left(1+2^{l}\left|a_{1}\left(t^{\prime}\right)\right|+\cdots+2^{l(Q-n)}\left|a_{Q-n}\left(t^{\prime}\right)\right|\right)^{-\alpha} d t^{\prime}
\end{gathered}
$$

which is bounded independent of $l$. The last inequality follows form [RS1] and the proof of the theorem is complete.

The general case where $\gamma$ is any connected analytic curve in $G$, follows by transference [CW, RS2] as is shown in the following remarks.

Remark. (vi) Assume now that $\gamma$ generates a proper subgroup of $G$. As in Remark (ii) we choose $\left\{e_{1}, \ldots, e_{l}\right\}$ that generate a Lie subalgebra $h$ of $g$. Extend it to a basis $\left\{e_{1}, \ldots, e_{l}, \ldots, e_{n}\right\}$ of $g$ and define for $r>0$, $D_{r}\left(x_{1}, \ldots, x_{l}, \ldots, x_{n}\right)=\left(r^{a_{1}} x_{1}, \ldots, r^{a_{l}} x_{l}, \ldots, r^{a_{n}} x_{n}\right)$ where $a_{1}, \ldots, a_{l}$ are determined by $\gamma$ and $a_{l+1}, \ldots, a_{n}$ are arbitrary integers. Assume that $D_{r}$ are automorphisms of $G$, let $\gamma_{0}$ be the homogeneous curve (w.r.t. $D_{n}$ ) associated to $\gamma$, and let $H$ be the subgroup of $G$ generated by $\gamma_{0}$.

Then $H$ is homogeneous with the dilations induced and $\gamma$ generates $H$. By transference, $T f=f * \mu$ is bounded on $L^{p}(G)$.

Remark. (vii) Let $\gamma$ be an analytic curve that generates a connected and simply connected, nilpotent Lie Group $N$. Let $D_{r}$ be a group of dilation associated to 
$\gamma$, which are not necessarily automorphisms of $N$ and let $\gamma_{0}$ be as in Remark (ii). As in [RS2] let $g$ be the step $m$ free Lie algebra generated by $n$, for $m$ large enough and let $G$ be the simply connected group with $\mathscr{L}(G)=g$. Then there exists $H$ a normal subgroup of $G$ such that $G / H$ is isomorphic to $N$. Denote by $\sigma: g \rightarrow n$ the quotient morphism. By construction of $g$ the dilations $D_{r}$ on $n$ extend to automorphisms $\widetilde{D}_{r}$ of $g$.

Let $\bar{\gamma}=\exp _{N}^{-1} \circ \gamma, \tilde{\gamma}=\exp _{G} \circ \bar{\gamma}$ and $\bar{\gamma}_{0}=\exp _{N}^{-1} \circ \gamma_{\circ}, \tilde{\gamma}_{\circ}=\exp _{G} \bar{\gamma}_{0}$. Then $\tilde{\gamma}_{\circ}$ is a homogeneous curve w.r.t. $\widetilde{D}_{r}$ and the coordinates of $\tilde{\gamma}_{\circ}$ (resp. $\tilde{\gamma}$ ) with respect to $\exp _{G}$ are those of $\gamma_{0}$ (resp. $\gamma$ ). Thus $T f=f * \mu$ is bounded on $L^{p}(G), 1<p<\infty$ and by transference on $L^{p}(N), 1<p<\infty$.

Remark. (viii) The maximal function along $\gamma$,

$$
M_{\gamma} f(x)=\sup _{r \leq 1} \frac{1}{r}\left|\int_{0 \leq|t| \leq r}\right| f\left(x \gamma(t)^{-1}\right) \mid d t
$$

is bounded on $L^{p}(G), 1<p \leq \infty$. Also

$$
T^{*} f(x)=\sup _{r}\left|\int_{r \leq|t| \leq 1} f\left(x \gamma(t)^{-1}\right) d t / t\right|
$$

is bounded on $L^{p}(G), 1<p<\infty$ and hence $T f(x)$ exists a.e. for all $f \in$ $L^{p}(G), 1<p<\infty$.

To see this we first remark that if $H f=\sup _{j}\left|f * f_{j}\right|$ with $f_{j}$ satisfying

(i) $\left\|f_{j}\right\|_{1} \leq \mathbf{C}_{1}$,

(ii) $\int\left|f_{j}(x y)-f_{j}(x)\right| \leq \mathbf{C}_{1} 2^{j \epsilon}|y|^{\epsilon} \forall \epsilon>0$,

(iii) $\sup f_{j} \subset\left\{|x| \leq \mathbf{C} 2^{-j}\right\}$.

Then $H$ is bounded on $L^{\infty}$ and of weak type (1.1) with constant $C_{1} C_{\epsilon}$. Indeed, we can assume that $f \geq 0$. For $\lambda>0$ we decompose $f=g+b$ where $\|g\|_{\infty} \leq \lambda, b=\sum_{j} b_{j}, \sup b_{j} \subset B_{j}=B\left(a_{j}, r_{j}\right), \int b_{j}=0, \int\left|b_{j}\right| \leq$ $A \lambda\left|B_{j}^{*}\right|$, and $\sum\left|B_{j}^{*}\right| \leq \frac{A}{\lambda}\|f\|_{1}, B_{j}^{*}=B\left(a_{j}, 2 r_{j}\right)$. Then it is enough to estimate $|\{x \mid H b(x)>\lambda\}|$. But

$$
\{x \mid H b(x)>\lambda\} \subset\left(\bigcup_{j} B_{j}^{*}\right) \bigcup\left\{x \in{ }^{c}\left(\bigcup_{j} B_{j}^{*}\right) \mid H b(x)>\lambda\right\}
$$


and

$$
\begin{aligned}
\mid\{x & \left.{ }^{c}\left(\bigcup B_{j}^{*}\right) \mid H b(x)>\lambda\right\} \mid \\
& \leq \frac{1}{\lambda} \int_{c_{\left(\cup B_{j}^{*}\right)}} H b(x) \leq \frac{1}{\lambda} \sum_{i} \int_{{ }^{c} B_{i}^{*}} H b_{i}(x) \\
& =\frac{1}{\lambda} \sum_{i} \int_{c_{B_{i}^{*}}} \sup _{j}\left|\int_{B_{i}} b_{i}(y) f_{j}\left(y^{-1} x\right) d y\right| d x \\
& =\frac{1}{\lambda} \sum_{i} \int_{c_{B_{i}^{*}}} \sup _{j} \mid \int_{B_{i}} b_{i}(y)\left(f_{j}\left(y^{-1} x\right)-f_{j}\left(a_{i}^{-1} x\right) d y \mid d x\right. \\
& \leq \frac{1}{\lambda} \sum_{i} \int_{B_{i}}\left|b_{i}(y)\right| \sum_{j} \int_{C_{B_{i}^{*}}}\left|f_{j}\left(y^{-1} x\right)-f_{j}\left(a_{i}^{-1} x\right)\right| d x d y \\
& \leq \frac{1}{\lambda} \sum_{i} \int_{B_{i}}\left|b_{i}(y)\right| \sum_{j} \int_{|z|>2|\tilde{y}|}\left|f_{j}\left(\tilde{y}^{-1} z\right)-f_{j}(z)\right| d z \\
& \leq \mathbf{C}_{\epsilon} \cdot \frac{1}{\lambda} \sum_{i} \int_{B_{i}}\left|b_{i}(y)\right| d y \quad \text { by (ii) and (iii), } \tilde{y}=y^{-1} a_{i}
\end{aligned}
$$

Now

$$
M_{\gamma} f(x) \leq \mathbf{C} \sup \left(f *\left|\mu_{j}\right|\right)(x)
$$

and

$$
\sup _{j}\left(f *\left|\mu_{j}\right|\right) \leq \sum_{k \geq 1} \sup _{j}\left|f * \eta_{k+j} *\right| \mu_{j}||+\sup _{j}\left(f * \varphi_{j} *\left|\mu_{j}\right|\right)=\sum_{k} \widetilde{M}_{k}+\tilde{N}
$$

By the above $\widetilde{M}_{k}$ is bounded on $L^{\infty}$ and for each $\epsilon>0$ there exists a constant $C_{\epsilon}$ such that $\widetilde{M}_{k}$ is of weak type (1.1) with constant $C_{\epsilon} 2^{k \epsilon}$. The same holds for $\tilde{N}$. We also have that $\left\|\widetilde{M}_{k}\right\|_{2,2} \leq \mathrm{C} 2^{-\sigma k}$ for some $\sigma>0$, by using the technique of the square functions (see [Ch]). Indeed we have to check that the operators $S_{k} f(x)=\sum_{j} a_{j} f * \eta_{k+j} *\left|\mu_{j}\right|, a_{j}= \pm 1 \forall j$, have $L^{2}$ boundedness $\mathrm{C} 2^{-\sigma k}$ but this follows as in the proof of the theorem since $n$-times $\left|\mu_{j}\right| * \cdots *\left|\mu_{j}\right|$ is absolutely continuous.

The boundedness of $T^{*}$ on $L^{p}, 1<p<\infty$, reduces to prove that the operators

$$
T_{1} f(x)=\sup _{i \geq 0}\left|f * \sum_{j \geq i} \mu_{j} * \varphi_{i}\right|
$$

and

$$
T_{2} f(x)=\sup _{i}\left|\sum_{j=0}^{i} f * \mu_{j} *\left(\delta-\varphi_{i}\right)\right|
$$

are bounded on $L^{p}$ for $1<p<\infty$ (see [Ch]). $T_{1} f(x)$ is bounded pointwise by the maximal operator on $G$ since the functions $\psi_{i}=\sum_{j>i} \mu_{j} * \varphi_{i}$ satisfy $\sup \psi_{i} \subset\left\{x|| x \mid \leq \mathbf{C} 2^{-i}\right\}$ and $\left|\psi_{i}(x)\right| \leq \mathbf{C} 2^{i Q}$. 
Now $T_{2} f(x) \leq \sum_{k} \sup _{j}\left|f * \mu_{j} *\left(\delta-\varphi_{k+j}\right)\right|=\sum_{k} \widetilde{M}_{k}$ and $\widetilde{M}_{k} f \leq$ $M_{\gamma} f+\sup _{j}\left|f * \mu_{j} * \varphi_{k+j}\right|$. Since $\sup _{j}\left|f * \mu_{j} * \varphi_{k+j}\right|$ is bounded on $L^{\infty}$ and of weak type (1.1), it is bounded on $L^{p}$ for $1<p<\infty$ and so is $\widetilde{M}_{k}$. To see that $\left\|\widetilde{M}_{k}\right\|_{2,2} \leq \mathbf{C} 2^{-\sigma k}$, for some $C, \sigma>0$, we can argue as in the theorem since $n$-times $\mu_{j} * \cdots * \mu_{j}$ is absolutely continuous with density $\rho_{j}$ satisfying the equation $\int\left|\rho_{j}(x y)-\rho_{j}(x)\right| \leq \mathbf{C} 2^{j \epsilon}|y|^{\epsilon}$ and $\left(\delta-\varphi_{k+j}\right)$ is a measure with bounded $L^{1}$-norm, $\operatorname{supp}\left(\delta-\varphi_{k+j}\right) \subset\left\{x|| x \mid \leq \mathbf{C} 2^{-(k+j)}\right\}$ and it has mean value zero.

\section{ACKNOWLEDGMENTS}

I am deeply indebted to Fulvio Ricci with whom I had the privilege to work at the Politecnico di Torino. He suggested this problem and I wish to express my gratitude for his generous help during its preparation.

Added in proof. I am very grateful to the referee for informing me of a recent preprint by M. Christ, A. Nagel, E. Stein, and S. Wainger [ChNSW] where more general results are presented, and from which our main theorem follows.

\section{REFERENCES}

[Ch] M. Christ, Hilbert transform along curves, I, Ann. of Math. 122 (1985).

[ChNSW] M. Christ, A. Nagel, E. Stein, and S. Wainger, Singular and maximal Radon transforms, preprint.

[CW] R. Coifman and G. Weiss, Analyse harmonique non-commutative sur certain espaces homogenes, Lecture Notes in Math., vol 242, Springer-Verlag, Berlin and New York, 1971.

[H] L. Hormander, An introduction to complex analysis in several variables, 2nd ed. NorthHolland Mathematical Library, vol. 7, 1973.

[RS1] F. Ricci and E. Stein, Harmonic analysis on nilpotent groups and singular integrals, Internat. J. Funct. Anal. 73 (1987).

[RS2] _ Harmonic analysis on nilpotent groups and singular integrals, II. J. Funct. Anal. 77 (1988).

[SW] E. Stein and S. Wainger, Problems in harmonic analysis related to curvature, Bull. Amer. Math. Soc. 84 (1978).

Fa. M.A.F., Valparaíso y Rogelio Martínez, Ciudad Universitaria 5000, Cordoba, ARGENTINA 